\title{
The First Awake Craniotomy for Eloquent Glioblastoma in Southern Thailand
}

Anukoon Kaewborisutsakul, M.D. ${ }^{1}$, Sakchai Sae-Heng, M.D. ${ }^{1}$, Chanatthee Kitsiripant, M.D. ${ }^{2}$ Pannawit Benjhawaleemas, M.D. ${ }^{2}$

${ }^{1}$ Neurological Surgery Unit, Department of Surgery, ${ }^{2}$ Department of Anesthesiology, Faculty of Medicine,

Prince of Songkla University, Hat Yai, Songkhla 90110, Thailand.

Received 10 September 2019 • Revised 31 October 2019 • Accepted 10 November 2019 • Published online 19 December 2019

\section{Abstract:}

Awake craniotomy (AC) with direct cortical stimulation is becoming the gold standard for functional brain mapping. It is used to identify the safe brain area before pathologic resection. This method indicates the pathology near or at the eloquent cortex, such as gliomas or metastasis. AC can optimize the patient's quality of life and oncologic outcome. This task requires the active cooperation of a patient care team familiar with advanced neuroscience and challenging to learn. We report the first time this operation which performed in our institute with technical details, in terms of anesthesia, and surgical aspects.

Keywords: awake craniotomy, brain tumor, glioblastoma

Contact: Anukoon Kaewborisutsakul, M.D.

Neurological Surgery Unit, Department of Surgery, Faculty of Medicine,

Prince of Songkla University, Hat Yai, Songkhla 90110, Thailand.

E-mail: anukoonkaew@gmail.com
J Health Sci Med Res 2020;38(1):61-65 doi: 10.31584/jhsmr.202077 www.jhsmr.org

(ㄷ 2020 JHSMR. Hosting by Prince of Songkla University. All rights reserved.

This is an open access article under the CC BY-NC-ND license

(http://www.jhsmr.org/index.php/jhsmr/about/editorialPolicies\#openAccessPolicy). 


\section{Introduction}

Awake craniotomy is a neurosurgical procedure for remove intracranial lesionthat is performed while patients are awake and alert. $^{1}$ This kind of surgery is helpful for the lesion located near the functional area, called the "eloquent cortex." Previous studies have demonstrated that it can decrease the postoperative neurological deficit and increase the volume of lesion resection., ${ }^{1,2}$ This procedure is indicated for intrinsic brain lesions, especially for gliomas (both low and high grades) and metastasis. ${ }^{2}$ However, the success of this operation requires appropriate patient selection, preparation, a specialized anesthetic technique, adequate intraoperative testing, and monitoring. ${ }^{3}$ We report the first case of awake craniotomy at our institute in June 2017. The case is presented following the Surgical Case Report guideline. ${ }^{4}$

\section{Case presentation}

The patient was a 60-year-old, right-handed man. He presented with progressive headache and dizziness for two weeks and a mild degree of weakness on the left. $\mathrm{He}$ had no underlying diseases and physical examination was unremarkable. The magnetic resonance imaging (MRI) showed a heterogeneous rim gadolinium-enhancing lesion at the right parieto-temporal lobe; mass volume was 47.6 milliliters $(\mathrm{ml})$, with surrounding brain edema and a midline shift to the left side of 1.2 centimeters (cm) (Figure 1). He was admitted to the neurosurgery ward and received 20 milligrams (mg) of dexamethasone per day and phenytoin for seizure prophylaxis Awake craniotomy was considered beneficial for this patient because his tumor was near the eloquent cortex. The patient received preoperative information about the risks and benefits of awake craniotomy, and underwent an operation on the fifth day after his admission.

\section{Patient preparation}

Patient preparation included disease explanation, management plan, and the steps of surgery and anesthesia. The patient rehearsed his intraoperative tasks before the date of the operation with a motor command, sensory examination, reading, and picture naming test with the commercial alphabet test. He also simulated the supine operative position and turned his head and body to the left side in this preparatory phase. On the day of surgery, the patient was premedicated with dexamethasone $10 \mathrm{mg}$ intravenously and phenytoin level was 7.6 milligrams per liter (mg/). He received an additional dose of $500 \mathrm{mg}$ of intravenous phenytoin before starting the operation.

\section{Intraoperative: anesthesia and cortical stimulation}

In the operating room, we selected the asleepawake-asleep method for anesthetic management. Firstly, basic anesthetic monitoring (blood pressure, pulse oximeter and electrocardiography), and additionally processed electroencephalography (BIS ${ }^{\mathrm{TM}}$ Medtronic Covidien, Dublin, Ireland) were applied. The proper sized nasal airway was used for assuring airway patency, and oxygen supplement was delivered via nasal cannula with EtCO2 monitoring. Dexmedetomidine $1 \mu \mathrm{g} / \mathrm{kg}$ was administered over 10 minutes as a loading dose, then followed by a maintenance infusion of 0.7 micrograms per kilogram per hour ( $\mu \mathrm{g} / \mathrm{kg} / \mathrm{hr})$. After the dexmedetomidine was started, a scalp block was performed on the right side with $20 \mathrm{ml}$ of $0.5 \%$ levobupivacaine. After that, the target-controlled infusion was started with Propofol according to Schnider's model between 1.5 and 2.5 micrograms per milliliter $(\mu \mathrm{g} / \mathrm{ml})$ to maintain BIS values 40 and 60 during the asleep period. Afterward, invasive blood pressure monitoring and another large-bore intravenous line were done. 

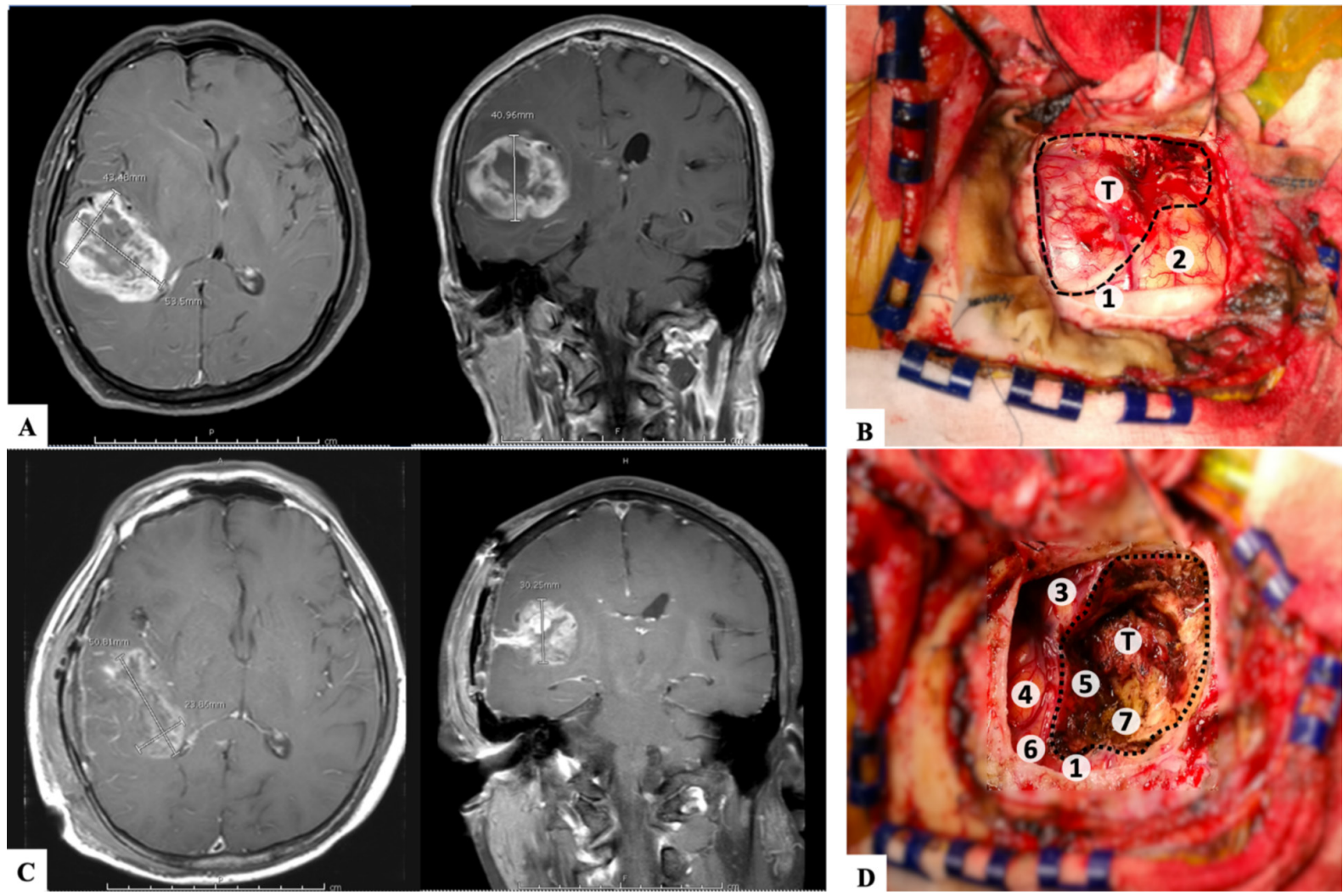

Figure 1 A Preoperative axial and coronal T1-weighted with gadolinium injected magnetic resonance imaging showing an enhancing tumor at right temporo-parietal lobe with calculated volume 47.6 milliliters. B Intraoperative finding before resection. Alphabet tag $(\mathrm{T})$ and dash line showing the boundary of tumor, which defined by intraoperative ultrasound. The number of tags related to the eloquent cortex: Tag 1, the primary somatosensory cortex, eliciting left face and lip dysesthesia Tag 2, suspected the inferior parietal lobule which found negative stimulation. C Postoperative day 3, axial, and coronal T1-weighted with gadolinium injected magnetic resonance imaging showing an enhancing residual tumor, calculated volume 18.3 milliliters. D Intraoperative finding after resection, dot line showing the boundary of the tumor resection cavity. Alphabet tag ( $T$ ) showing the remaining tumor. Tag 3, the superior temporal gyrus Tag 4 and 5, the primary motor cortex, eliciting left face and finger movement Tag 1 and 6, the primary somatosensory cortex and Tag 7, suspected subcortical thalamocortical fibers, eliciting left face and lip dysesthesia.

The scalp was infiltrated with $6 \mathrm{ml}$ of $1.0 \%$ xylocaine with 1:200,000 adrenaline (2 $\mathrm{ml}$ per site) before placement of the three-point Mayfieldo head clamp and further 20 $\mathrm{ml}$ of $1.0 \%$ xylocaine with $1: 200,000$ adrenaline along the incision. After skull removal step, the dura was injected with $1.0 \%$ xylocaine with $1: 200,000$ adrenaline via 30 gauze needle and a small dose of intravenous fentanyl was used as an adjunct when the blood pressure increased more than $20.0 \%$ from baseline. Twenty minutes after dexmedetomidine and Propofol discontinuation, the 
patient became fully awake. The tumor was located by using intraoperative ultrasonography. Intraoperative electrocorticography was monitored. The rehearsing tasks (picture naming with or without simultaneous contralateral arm movement) were performed before electrical stimulation, upon which the entire resection phase was reassessed; both cortical and subcortical mapping. The direct cortical stimulation used a 5 millimeters $(\mathrm{mm})$ space tip of the bipolar probe (Nim eclipse ${ }^{\circledR}$, Metronic, USA). The setting of biphasic current was pulse frequency $60 \mathrm{Hertz}(\mathrm{Hz})$, pulse duration of 1 millisecond (msec), amplitude 1.5 to 12 milliampere $(\mathrm{mA})$, and duration of the stimulation was up to 4 seconds. The current amplitude was adjusted with a progressive fashion in the step of $0.5 \mathrm{~mA}$ until a response was detected. Indeed, the cortex that did not show the specific response to stimulation three consecutive times was defined as the negative area. For this patient, the positive stimulation was detected on the anterior aspect of the tumor with dysesthesia and tonic face and hand movement, as defined as the primary sensorimotor cortex. Intraoperative seizure detected by after discharge signal and briefly following with a focal seizure on his arm for a few seconds during cortical stimulation with amplitude $12 \mathrm{~mA}$. Cold saline was irrigated and the operation was paused for around 5 minutes after stop clinical seizure and normalized electroencephalography. The partially tumor resection was stopped due to the patient very anxious after seizure and complained severe dysesthesia along his face and lip. The medio-inferior part of the tumor is remained. After dura closure by using water-tight technique, conscious sedation was provided with dexmedetomidine for skin closure. During the operation, the systolic blood pressure was maintained in the range of $100-150 \mathrm{mmHg}$, heart rate of $52-60$ beats/min, peripheral oxygen saturation of $98.0-100.0 \%$, and endtidal carbon dioxide (CO2) of $26-36 \mathrm{mmHg}$, and BIS value of 40-60. The duration of the operation was 8 hours.

\section{Patient's outcome}

The postoperative period was uneventful and the patient was discharged from the institute on the postoperative day 7 . The extent of resection, $61.5 \%$, was demonstrated on postoperative MRI (Figure 1). Pathological study reported glioblastoma multiforme, then the patient received postoperative concurrent chemoradiation, the standard Stupp's regimen. Eight months after the operation his tumor had progressed and thus he underwent craniotomy under general anesthesiadue to profound weakness. After that, his clinical condition rapidly deteriorated into dependent status. Regretfully, he died due to septic shock at 14 months from his diagnosis.

\section{Discussion}

Awake craniotomy is a neurosurgical operation that was developed for the patient safety. Its benefits provide minimizing the new postoperative neurological deficits, while maximizing adequate lesionectomy.The most common indication for this procedure is an intrinsic brain tumor, especially gliomas near the eloquent cortex as presented in this case., ${ }^{4,5}$ To date, a growing body of evidence supports the concept of maximizing safe resections while balancingbetweenthe extent of resection and postoperative quality of life. ${ }^{6}$ Hence, All steps of surgery and criticalevents, such as intraoperative seizure,were reconsidered for achieved the best of care in the future.

In this case, one episode of intraoperative seizure (IOS) was detected during cortical stimulation. Previous reports reveal IOS as one of the most common complications of awake craniotomy. Its incidence ranges from $2.2-21.5 \%$. $^{6,7}$ It is also the leading cause of failed awake operations and related to poor postoperative outcomes. ${ }^{6,7}$ To reduce the incidence of IOS, the existing evidence still posessome unresolved questions. First, the role of preoperative antiepileptic drugs (AEDs) in patients with 
no preoperative seizures is inconclusive. The Japanese guideline for awake craniotomy recommends initiating the AEDs in advance to achieve a sufficient plasma drug concentration $^{3}$, whilst some European centers do not use the AEDs in a prophylaxis role. ${ }^{7}$ Second, the stimulation parameter varies among centers, such as frequencies, pulse phasic, and intensity, which found an association between stimulation intensity and IOS rate in the linear fashion. ${ }^{8}$ Generally, the intensity of bipolar stimulation does not exceed $20 \mathrm{~mA}$; however, one report showed that the maximum stimulation current of $4 \mathrm{mAis}$ adequate for creating the stimulation's effect with a low rate of 10 S. $^{9}$

After this case, we regularly perform awake craniotomy in almost all cases ofintrinsic supratentorial lesion near the eloquent cortex.

\section{Conclusion}

Despite lack of experience, the first case of awake craniotomy can be carried out safely and successfully, if it is well planned, prepared and organized.Team communication and preparation are the cornerstones of success.

\section{Acknowledgement}

The authors are thankful to our patient's care team, especially Mrs. Varunee Buntuchai, the head scrub nurse of the neurosurgical operating room, Songklanagarind Hospital. We also thank to the Office of International Affairs for edited and revised language in manuscript.

\section{Conflict of interest}

The authors have declared that no conflict of interests exist.

\section{References}

1. Sanai N, Mirzadeh Z, Berger MS. Functional outcome after language mapping for glioma resection. N Engl J Med 2008; 358:18-27.

2. Paldor I, Drummond KJ, Awad M, Sufaro YZ, Kaye AH. Is a wake-up call in order? Review of the evidence for awake craniotomy. J ClinNeurosci 2016;23:1-7.

3. Kayama T. The guidelines for awake craniotomy guidelines committee of the Japan awake surgery conference. Neurol Med Chir (Tokyo) 2012;52:119-41.

4. Agha RA, Borrelli MR, Farwana R, Koshy K, Fowler AJ, Orgill DP, et al. The SCARE 2018 statement: updating consensus Surgical CAse REport (SCARE) guidelines. Int $\mathrm{J}$ Surg 2018;60:132-6

5. De Witt Hamer PC, Robles SG, Zwinderman AH, Duffau H, Berger MS. Impact of intraoperative stimulation brain mapping on glioma surgery outcome: a meta-analysis. J Clin Oncol 2012;30:2559-65.

6. Hervey-Jumper SL, Berger MS. Maximizing safe resection of low- and high-grade glioma. J Neurooncol 2016;130:26982.

7. Hervey-Jumper SL, Li J, Lau D, Molinaro AM, Perry DW, Meng L, et al. Awake craniotomy to maximize glioma resection: methods and technical nuances over a 27-year period. J Neurosurg 2015;123:325-39.

8. Szelényi A, Bello L, Duffau H, Fava E, Feigl GC, Galanda M, et al. Intraoperative electrical stimulation in awake craniotomy: methodological aspects of current practice. Neurosurg Focus 2010;28:E7.

9. Boetto J, Bertram L, Moulinié G, Herbet G, Moritz-Gasser S, Duffau $\mathrm{H}$. Low rate of intraoperative seizures during awake craniotomy in a prospective cohort with 374 supratentorial brain lesions: electrocorticography is not mandatory. World Neurosurg 2015;84:1838-44. 